\title{
When thrombocytopenia can lead to fetal stroke?
}

\author{
Magdalena Sandu', Ileana Barascu², Catalin Carstoveanu², Raluca Teleanu' ${ }^{1}$ \\ ${ }^{1}$ Pediatric Neurology Department, "Dr. Victor Gomoiu" Children's Hospital, \\ "Carol Davila" University of Medicine and Pharmacy, Bucharest, Romania \\ ${ }^{2}$ Neonatal Intensive Care Unit, "Marie S. Curie" Children's Emergency Hospital, \\ "Carol Davila" University of Medicine and Pharmacy, Bucharest, Romania
}

\begin{abstract}
Fetal stroke is a rare disease that occurs between 14 weeks of gestation and the onset of labor and can be caused by ischemic, thrombotic or hemorrhagic injury produced during pregnancy. One of the maternal conditions associated with fetal stroke is alloimmune thrombocytopenia. This needs to be differentiated from autoimmune thrombocytopenia, a condition in which mothers are previously affected. The neurological consequences of the fetal brain injury may be delayed neurological milestones, cognitive impairment, epilepsy and cortical blindness. Magnetic resonance represents the best diagnostic method to reveal prenatal brain injury.
\end{abstract}

Keywords: fetal stroke, alloimmune/autoimune thrombocytopenia, intracranial hemorrhage, neurological consequences

\section{INTRODUCTION}

Stroke represents a focal or multifocal brain lesion, with an underlying vascular cause, which can appear even during pregnancy, affecting the fetal cerebral development. This has been increasingly recognized in the last decade, along with the progress of neuroradiology methods and raised many questions about its causes. In spite of medical efforts, in approximately half of the cases (1), the physiopathological mechanism for fetal stroke remains unclear.

Alloimune thrombocytopenia represents a rare condition due to the transplacental passage of maternal antibodies against fetal platelet antigens, which are paternally derived. This passage starts at 14 weeks of gestation and fetal glycoproteins may begin to express on platelets as early as 16 weeks, when the symptoms may appear. It can be considered an equivalent of red blood cells immunization and may lead to devastating manifestations during pregnancy, being known as the most common cause of intracranial hemorrhage (ICH) in fetal/neonatal period. The reported incidence of alloimune thrombocytopenia is 1:1,000-1,500 live births (2). Usually, mothers are asymptomatic with a normal platelet count and function, but a previous child with ICH represents a risk, especially at an early gestational stage. Maternal antibody levels correlate with neonatal platelet counts and if the level of anti HPA 1a (HPA-human platelet antigens) exceeds more than $3.0 \mathrm{IU} / \mathrm{ml}$ in either week 22 or 34 of gestation, the risk of severe fetal thrombocytopenia is high (3). After the diagnosis is made, a multidisciplinary team - obstetrician, gynecologist, hematologist, neonatologist, radiologist, neurosurgeon has to establish a treatment strategy with serial percutaneous umbilical blood sampling, intravenous immunoglobulin administration, corticotherapy, fetal platelet transfusions. The team needs to determine the best timing for delivery. The risk of $\mathrm{ICH}$ in the fetus appears to be low, if the platelet count does not fall under $20,000 / \mathrm{mm}^{3}$. Vaginal delivery cannot be recommended if the fetal platelet count is less than $50,000-100,000 / \mu \mathrm{L}$ (4) and once the birth was uneventful, the thrombocytopenia can 
resolve spontaneously, in 1 or 2 weeks, the platelets half-life being 8-10 days.

When antibodies affect both maternal and fetal platelets, the condition is called autoimune thrombocytopenia, usually due to maternal diseases, such as idiopathic thrombocytopenic purpura, systemic lupus erythematosus or hyperthyroidism. This is considered to be a milder condition than alloimune thrombocytopenia, both for mother and fetus, the risk of developing ICH being considerably lower (5).

\section{CASE REPORT}

A five months old infant was admitted to our hospital due to paroxistic movements and delay in neuropsychomotor development. The child was born from a pathological pregnancy. The mother was 36 years old, being treated for severe cortico-resistant, autoimmune thrombocytopenia. At the age of 28 , she was firstly diagnosed with thrombocytopenia during her first pregnancy, which needed to be terminated due to the high risk of hemorrhagic event at that time. She continued to be monitored in the hematological department and when medical treatment failed to control the disease, she underwent a splenectomy, with a favorable outcome, the platelet count stabilizing at $160,000 / \mathrm{mm}^{3}$.

Our patient, a boy, is her firstborn, prematurely, 2,500 $\mathrm{g}$ at gestational age of 32-33 weeks, under a cesarean section. While still in utero, he was first suspected (Fig. 1 Fetal US first trimester shows discreet enlargement of the right lateral ventricle) and then diagnosed with hemorrhagic stroke and secondary hydrocephalus (Fig. 2 Fetal MRI at 32 weeks of gestation shows enlargements of the ventricular system [a] and large porencefalic cyst in the right cerebral hemisphere [b]. The technical quality of the im-

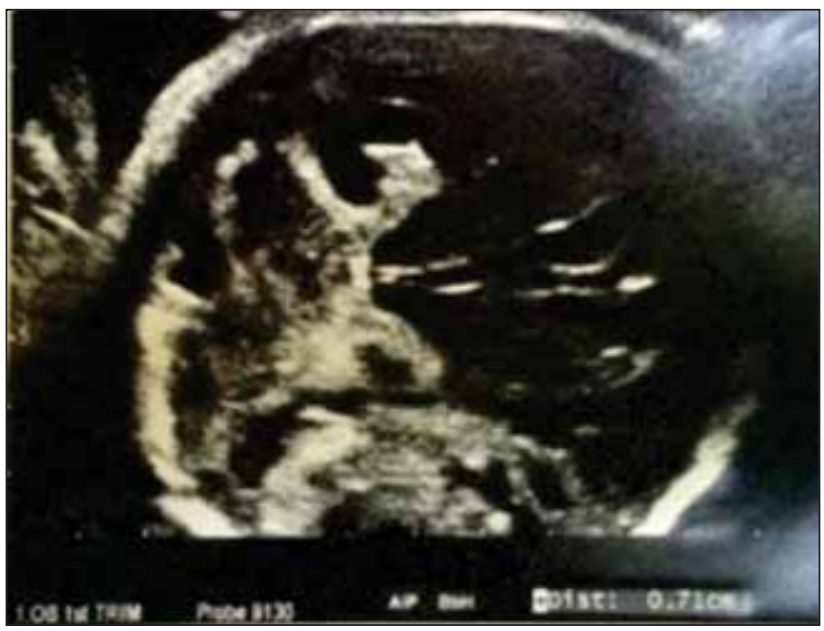

FIGURE 1. Fetal US first trimester shows discreet enlargement of the right lateral ventricle ages is not satisfying due to motion artefacts. The radiologist could not perform the appropiate fetal sequences, because of technical limitations).
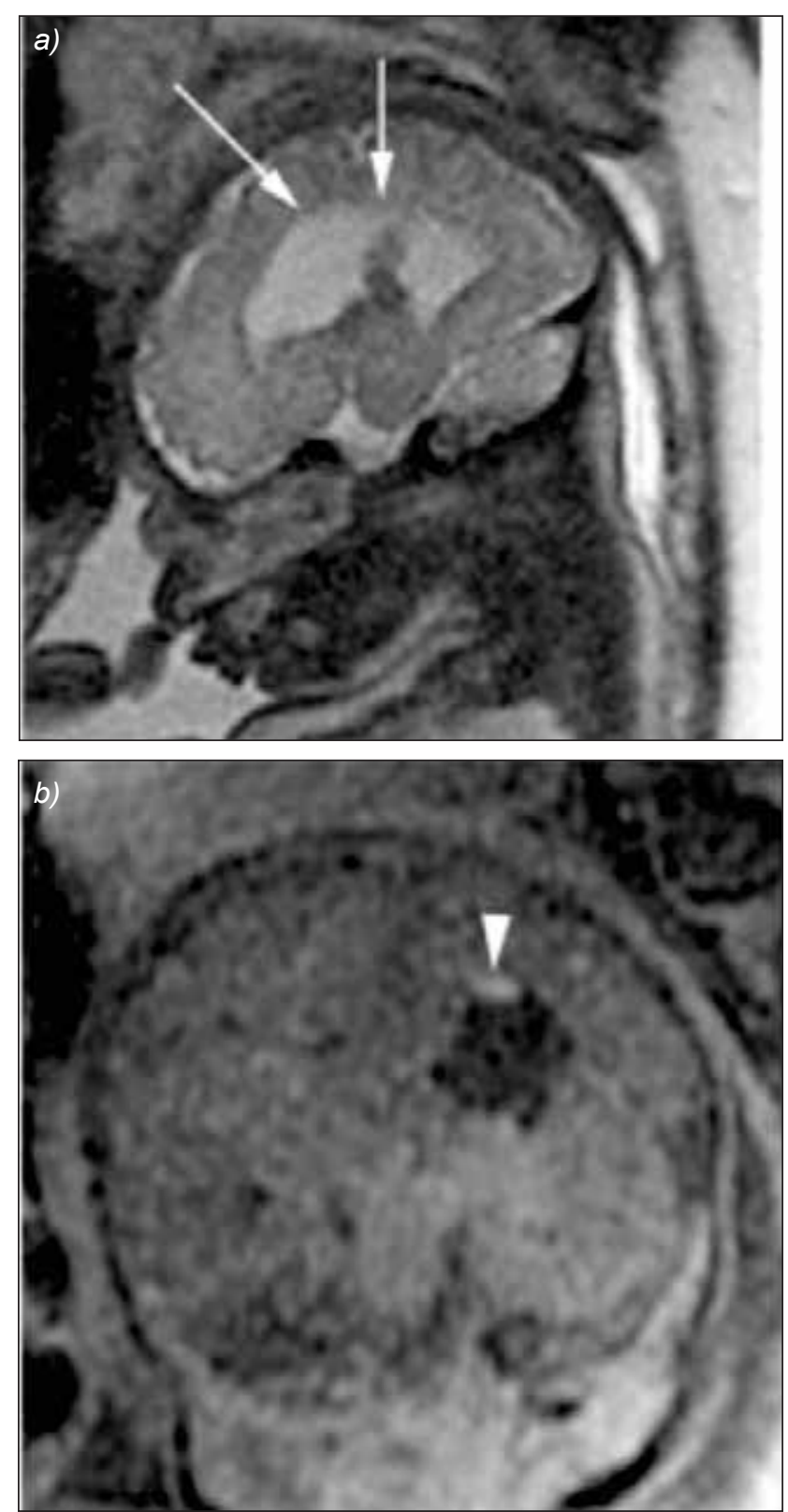

FIGURE 2. Fetal MRI at 32 weeks of gestation shows enlargements of the ventricular system (a) and large porencefalic cyst in the right cerebral hemisphere (b). The technical quality of the images is not satisfying due to motion artefacts. The radiologist could not perform the appropiate fetal sequences, because of technical limitations.

Postdelivery, the patient shows apnea, generalized hypotonia, pale cyanotic skin, micro petechias and generalized oedemas. Apgar Score was 5 at 1 minute and 7 at 5 minutes. Resuscitation maneuvers were required, including positive pressure ventilation (PPV) and continuous positive airway pressure (CPAP). He had a difficult postnatal adaptation, being oxygen dependent, showing progressive polyp- 
nea and severe progressive respiratory deficiency, maintaining moderate hypotonia and signs of mucocutaneous bleeding. He was intubated and placed into neonatal intensive care unit (NICU), where umbilical vessels were catheterized and he received hemostatic and antibiotic medication. Lab investigation reports have shown, immediately after birth, severe thrombocytopenia $\left(9,000 / \mathrm{mm}^{3}\right)$ and he received intravenous gamma globulins and dexamethasone along with blood transfusions (4 in the first 5 days). Antiplatelet antibodies were positive, the diagnostic method being only qualitative.

In spite of this treatment, the platelet count remained unstable, finally reaching normal levels at 2 months of age. Brestfeeding was stopped due to the suspicion that $\operatorname{IgA}$ antiplatelet antibodies coming from the mother may prolong neonatal thrombocytopenia (6). During relapses of thrombocytopenia, the newborn showed further signs of digestive, pulmonary and new cerebral bleedings. After birth, cerebral ultrasound revealed hyperechogenic and non-homogeneous large $(30 / 50 \mathrm{~mm})$ cyst in the parietal region of right cerebral hemisphere and asymmetric ventriculomegaly (left lateral ventricle bigger than right lateral ventricle). At the age of 6 weeks, the porencephalic cyst had grown from $30 / 50 \mathrm{~mm}$, to $40 / 64 \mathrm{~mm}$ and the head circumference had grown from $33 \mathrm{~cm}$ to $36.5 \mathrm{~cm}$. At the age of 2 months, cerebral tomography was performed (Fig. 3 a,b Large fluid collections - red arrow - in the right fronto-temporo-parietal-occipital region. Cerebral parenchimal tissue has reduced volume green arrow- and white matter appears hipointense, signaling encephalomacia) and a ventriculo-peritoneal shunt was required, in order to treat post hemorrhagic hydrocephalus (Fig. 4 Cerebral US after ventriculo-peritoneal shunt insertion; enlarged ventricles).

He was admitted to our clinic where neurological examination at 5 months revealed that the head circumference was $38.5 \mathrm{~cm}$, an anterior fontanelle of $0.5 / 0.5 \mathrm{~cm}$, normotensive, without control of the head, nystagmus, abnormal eye movements, global but asymmetrical spasticity, increased on the right hemibody, numerous, distonic postures affecting upper limbs and shoulders, clonoid reflexes. He was severely delayed from the neurological perspective and epilepsy was suspected.

He had long-term EEG (electroencephalographic) monitoring while awake (Fig. 5 Awake EEG dismorphic background with multifocal epileptiform discharges. Muscle artefacts at $\mathrm{Fp} 1$ ) and during sleep (Fig. 6 Sleep EEG showing lack of sleep spindles, multifocal discharges predominant-
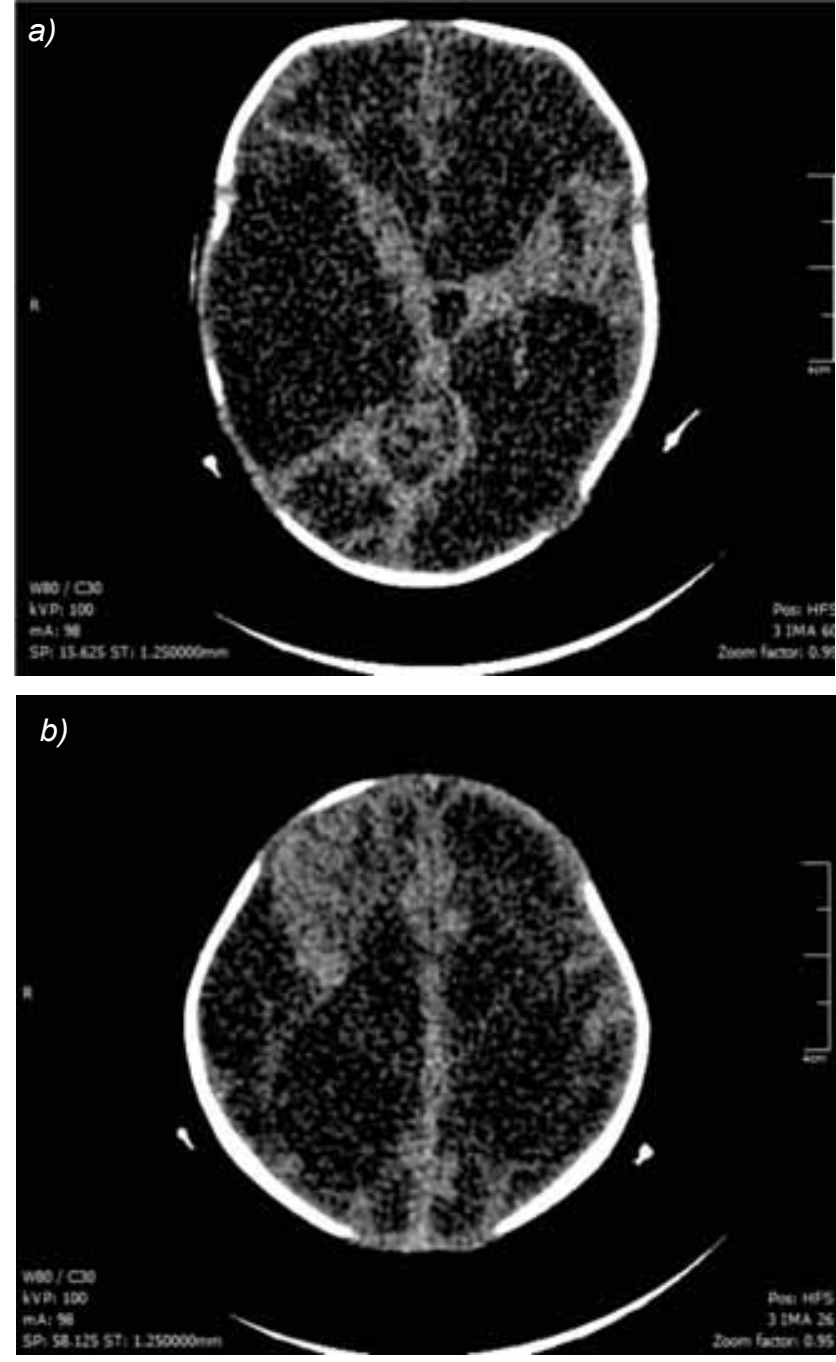

FIGURE 3. a,b Large fluid colections - red arrow - in the right fronto-temporo-parietal-occipital region. Cerebral parenchimal tissue has reduced volume - green arrow and white matter appears hipointense, signaling encephalomacia

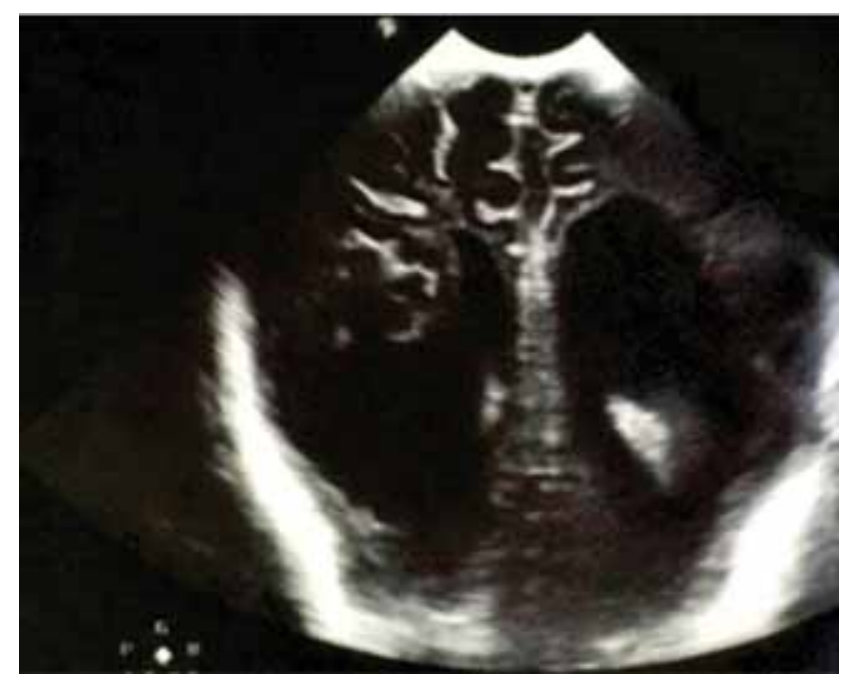

FIGURE 4. Cerebral US after ventriculo-peritoneal shunt insertion; enlarged ventricles

ly on the right hemisphere), which revealed some of the distonic postures and eye movements as pos- 


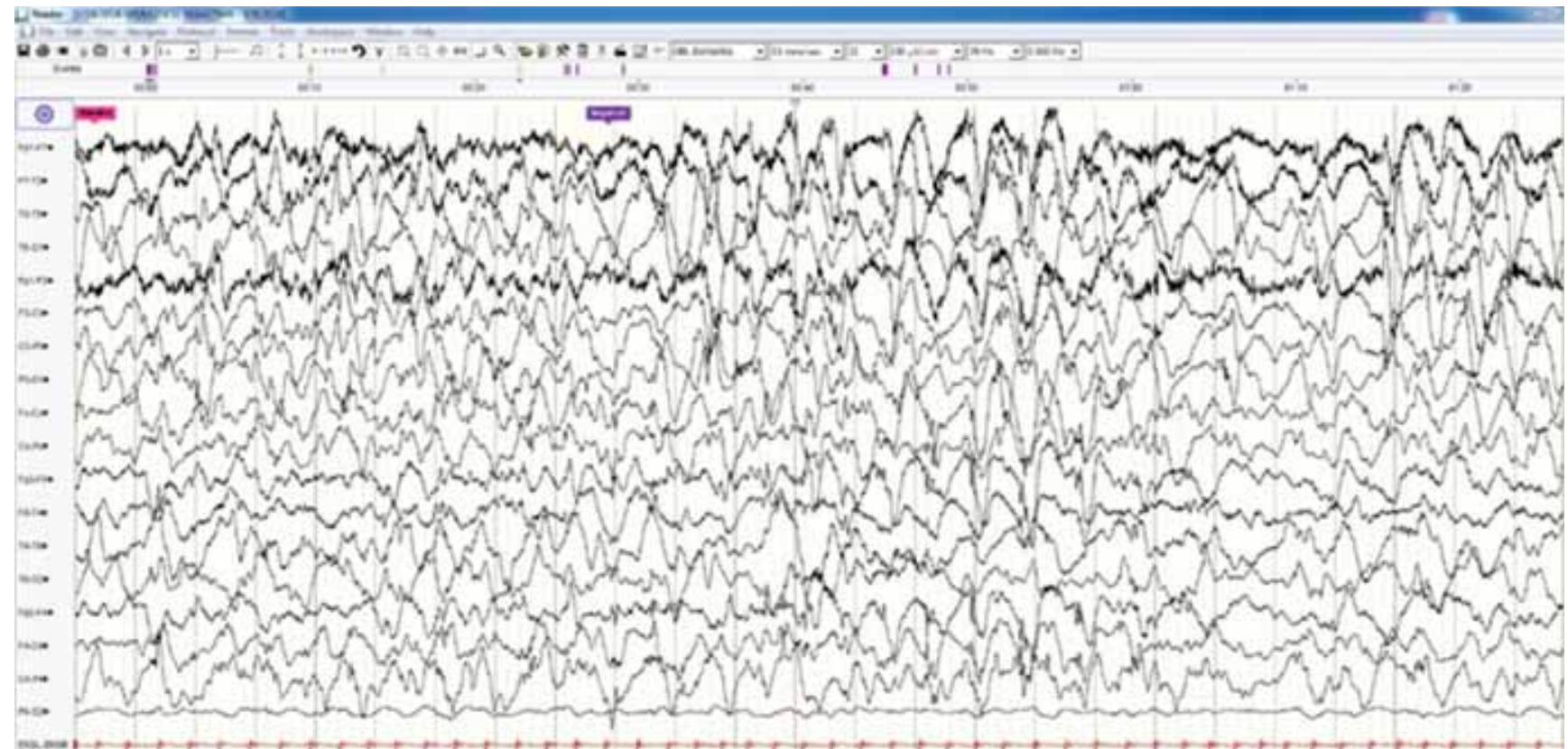

FIGURE 5. Awake EEG- dismorphic background with multifocal epileptiform discharges. Muscle artefacts at Fp1.

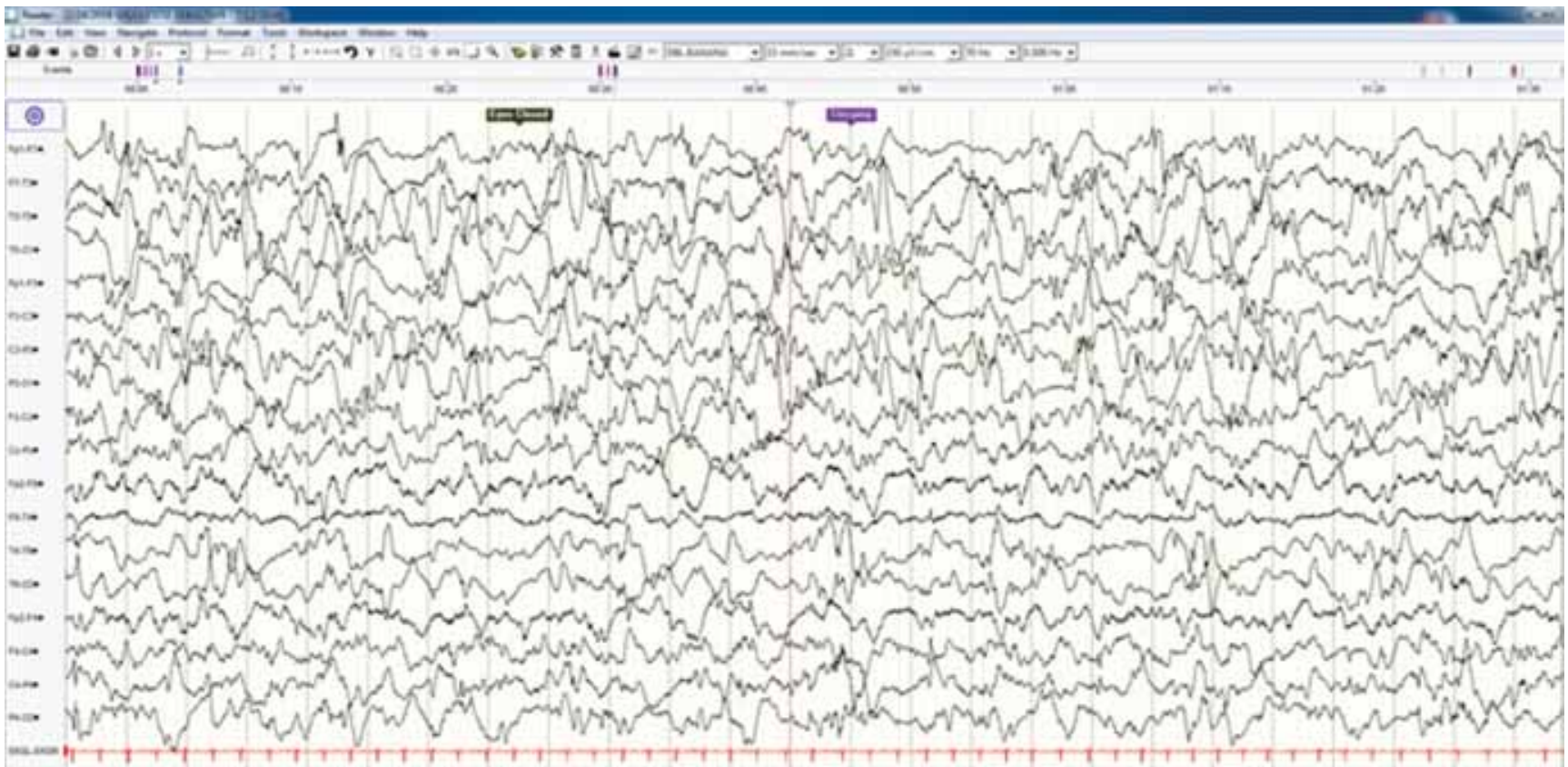

FIGURE 6. Sleep EEG showing lack of sleep spindles, multifocal discharges predominantly on the right hemisphere

sible ictal epileptic events. Levetiracetam was initiated and increased to $50 \mathrm{mg} / \mathrm{kg} /$ day, resulting in a lower frequency of abnormal postures and eye movement. However, the present clinical picture has a complex background (multifocal cerebral lesion, possible gastroesophageal reflux, retinopathy of prematurity).

At the moment, he is evaluated constantly by a multidisciplinary team - pediatric neurologist, pediatrician, ophthalmologist, neurosurgeon, physical medicine and rehabilitation doctor - in order to maximize the child's potential to achieve developmental milestones.

\section{CONCLUSION}

We have analyzed a case of severe neurological impairment caused by an ICH during fetal period. At a first glance, the most probable cause for fetal stroke in our case was autoimmune thrombocytopenia, due to the mother's previous condition, but the medical literature on this topic demonstrates that alloimune thrombocytopenia has, by far, a greater potential for $\mathrm{ICH}$. We believe that, during the fetal period, a complex mechanism acted, possibly combined, of autoimmune and alloimune thrombocytopenia. After birth, this condition was 
potentially worsened by breastfeeding, leading in the end to the clinical picture of a severely affected child. The case management was very difficult, requiring an extended medical team, long term hospi-

\section{REFERENCES}

1. Özduman K., Pober B.R., Barnes P., Copel J.A., Ogle E.A., Duncan C.C, et al. Fetal stroke. Pediatr Neurol. 2004; 30(3):151-62.

2. Sainio S., Järvenpää A.L., Renlund M., Riikonen S., Teramo K., Kekomäki R. Thrombocytopenia in Term Infants: A Population-Based Study. Obstet Gynecol. 2000; 95(3):441-6.

3. Killie M.K., Husebekk A., Kjeldsen-Kragh J., Skogen B. A prospective study of maternal anti-HPA 1a antibody level as a potential predictor of alloimmune thrombocytopenia in the newborn. Haematologica. 2008; 93(6):870-7. talization, overcoming lab limitations. This kind of pathology needs to be transferred to the closest NICU capable of performing the appropriate therapeutic intervention.

4. Mella M.T. Neonatal alloimmune thrombocytopenia. 2015;

5. Bussel J.B. Immune thrombocytopenia in pregnancy: autoimmune and alloimmune. J Reprod Immunol. 1997; 37(1):35-61.

6. Hauschner H., Rosenberg N., Seligsohn U., Mendelsohn R., Simmonds A., Shiff Y., et al. Persistent neonatal thrombocytopenia can be caused by IgA antiplatelet antibodies in breast milk of immune thrombocytopenic mothers. Blood. 2015; 126(5):661-4.

Conflict of interest: none declared Financial support: none declared 